\title{
Evaluation of Malaria Microscopy Diagnosis Performance in Public Hospitals of Eastern and Central Part of Oromia Region, Ethiopia, 2019
}

This article was published in the following Dove Press journal: Pathology and Laboratory Medicine International

\author{
Fraol Jaleta' \\ Getinet Garoma ${ }^{2}$ \\ Tadesse Gerenfes ${ }^{3}$ \\ 'Department of Research and Public \\ Health Emergency Preparedness, Adama \\ Public Health Research and Referral \\ Laboratory Centre, Adama, Ethiopia; \\ ${ }^{2}$ Department of Capacity Building and \\ Quality Assurance, Adama Public Health \\ Research and Referral Laboratory \\ Centre, Adama, Ethiopia; ${ }^{3}$ Department of \\ Molecular Biology, Adama Public Health \\ Research and Referral Laboratory \\ Centre, Adama, Ethiopia
}

Background: Prompt and accurate malaria diagnosis is an essential strategy for effective malaria case management as well as the public health response to malaria. Diagnosis based on clinical grounds alone may lead to misdiagnosis and mistreatment. The objective of this study is to evaluate the performance of malaria microscopy diagnosis in public hospitals of Eastern and Central part Oromia, Ethiopia.

Methods: A hospital-based cross-sectional study was conducted from March 2019 to May 2019 in 46 public hospitals of Eastern and Central parts of the Oromia region. Data were collected using structured checklists and pre-prepared known validated positive and negative slides. It was categorized and cleaned by Epi Info version 3.5.1 and analyzed by SPSS version 20 to identify factors associated with poor malaria microscopy diagnosis. The level of agreement was calculated by kappa statistics.

Results: From the total of 46 hospitals, 31 (67.39\%) had an acceptable quality performance with a cumulative grading score of $>80 \%$. The overall percentage of agreement in detection and species identification was $80.45 \%(\mathrm{kappa}=0.79)$ and $63.03 \%(\mathrm{kappa}=0.38)$. Laboratory professionals who had work experience greater than five years were 10.56 times better in detecting and identifying malaria parasite when compared to those who had work experience less than or equal to five years (AOR $[95 \% \mathrm{CI}]=10.56[1.45-76.73])$. Laboratory professionals who were trained in malaria microscopy diagnosis were 6.12 times reported better quality results than those who were not trained (AOR, \% 95CI=6.12 [1.5-48.13]).

Conclusion: The overall agreement of laboratory professionals in detection and species identification was with had substantial and fair agreement with kappa values of 0.79 and 0.38 , respectively. Lack of training and low work experience of laboratory professionals were factors associated with malaria microscopy diagnostic performance. Hence, capacitating laboratory professionals is essential to ensure good performance of malaria microscopy which reduces misdiagnosis of malaria parasites and mistreatment of malaria suspected patients.

Keywords: malaria diagnosis, level of agreement, mistreatment, Oromia

\section{Background}

Malaria is caused by protozoan parasites of the genus Plasmodium inoculated into the human host by a feeding female anopheline mosquito.

Prompt and accurate malaria diagnosis is essential for effective malaria case management as well as for public health response to malaria. Diagnosis based on clinical grounds alone may lead to misdiagnosis and mistreatment. Hence, accurate, reliable, and timely parasite-based malaria diagnosis is the only way to prevent and control malaria. ${ }^{1,2}$ 
Light microscopy remains the gold standard for species differentiation, parasite quantification, management of severe disease, and investigating treatment failures. External Quality Assessment (EQA) allows participant laboratories to assess their capabilities by comparing their results with other laboratories in the network; this can be achieved through panel testing (PT), blinded rechecking, and review of laboratory performance by onsite supervision. ${ }^{3}$

Routine parasitological confirmation of malaria is based on either light microscopy or detection of parasite antigens with rapid diagnostic tests. Quantitative buffy coat and polymerase chain reaction are also used in certain situations. ${ }^{4}$

Despite being preventable and treatable, malaria continues to have a devastating impact on people's health and livelihoods around the world. According to the World Health Organization (WHO) report of 2018, an estimated 219 million cases of malaria occurred worldwide in 2017 with estimated deaths of 435 . Of all malaria deaths, $93 \%$ were in the Sub-Saharan African Region. Plasmodium falciparum, which is the most dangerous malaria parasite, accounted for $99 \%$ of estimated malaria cases. ${ }^{5-7}$

Ethiopia has scaled up testing throughout the public health service system. According to the FMOH report of 2013, there were 3,331,599 confirmed cases of which $40 \%$ were diagnosed using microscopy. Plasmodium falciparum and Plasmodium vivax accounted for an average of $60 \%$ and $40 \%$ cases, respectively, from 2001 to 2016 in Ethiopia, and were the most dominant malaria parasites in all malaria-endemic areas. ${ }^{11,12}$

Accurate early diagnosis and prompt treatment of malaria are important components of one of the methods to prevent and control malaria in Ethiopia. Delay in diagnosis and inappropriate treatment of uncomplicated malaria, especially in children less than five years of age, pregnant women, and nonimmune individuals can lead to the rapid development of severe/complicated malaria. ${ }^{8,9}$

Misdiagnosis of malaria will result in the unnecessary prescription of high-cost drugs, the unnecessary exposure of the patient to potentially toxic drugs, and untreated malaria patients which leads to potentially severe consequences. Hence, correct laboratory diagnosis is essential in preventing false positive and false negative results which are equally problematic in patients with suspected malaria. Internal quality control (IQC) is a part of the Quality Assurance (QA) program and must be done regularly to ensure that all tests are performed accurately and precisely. ${ }^{13,16}$

Validation of slides read allows for monitoring of the performance of laboratory professionals over time and detection of additional problems, such as poor staining, poor slide preparation, and inadequate supplies and equipment. The currently recommended practical method is regular evaluation of the proportion of agreement between the first reading and expert re-examination of a minimum of 10 slides monthly. ${ }^{17}$

The greatest limitation to ensuring access to early diagnosis and effective treatment in malaria case management is the lack of adequate numbers, qualifications, and distribution of human resources including laboratory professionals who perform microscopy for diagnosis of malaria. Skilled laboratory professionals are essential for both effective diagnostic services and disease surveillance, which rely on their technical skills. ${ }^{18}$

Implementation of external quality assessment, which includes proficiency testing, onsite supervision, and blind rechecking, is essential to build and maintain the quality of malaria microscopy diagnosis. Sustaining good laboratory practice is one of the greatest challenges in building quality laboratory systems that produce accurate, reliable, and timely results in Ethiopia. Therefore, this study was aimed to evaluate the performance of malaria microscopy diagnosis and factors affecting its quality in public hospitals of Eastern and Central parts of Oromia, Ethiopia.

External Quality Assessment Scheme surveys indicate that there are continuing problems in malaria diagnosis such as inaccurate calculation of parasitemia or failure to estimate it altogether, difficulty in distinguishing between plasmodium species, and reporting the presence of malaria parasites when they were not actually present. ${ }^{10}$

\section{Materials and Methods}

\section{Study Setting and Material}

A hospital-based cross-sectional study was conducted from March 2019 to May 2019 in Eastern and central parts of Oromia which includes 12 zones. Eastern and central parts of the Oromia region have 50 public hospitals, 843 public health centers and one public health research and referral laboratory center providing healthcare services for the communities. ${ }^{19}$ All hospitals and health centers widely provide several clinical and diagnostic services including malaria microscopy diagnosis. They are supported, mentored, and supervised by Adama Public Health Research and Referral Laboratory Center to implement a laboratory quality management system program which ensures accurate and reliable test results for the patients. The study was conducted in all public hospitals which provide malaria microscopy diagnostic service during data collection. Likewise, laboratory professionals 
were randomly selected from each hospital of 46 public hospitals that were included in the study.

\section{Data Collection Tools and Technique}

Specimen slides for proficiency tests were prepared, standardized, and validated at Ethiopian Public Health Laboratory Association (EPHLA) and President's Malaria Institute (PMI) in Ethiopia, and the origin of the specimens used in this study was from confirmed positive and negative clinical leftover specimens. Data were collected after the checklist was prepared and validated according to the malaria national guidelines. Ten panel slides with known negative and positive results with different plasmodium species and stages were blindly distributed to each hospital. Laboratory professionals read the distributed slides as routine blood film examinations in accordance with the national recommended time required for each slide. A semi-structured questionnaire which comprises socio-demographic characteristics of laboratory professionals, quality assurance-related characteristics, and PT slide checklist was prepared from WHO and national guidelines to evaluate the overall performance of malaria microscopy diagnosis.

The lab professionals were also interviewed about the overall malaria microscopy diagnosis activities and laboratory performance was evaluated using a checklist prepared from WHO and national guidelines.

\section{Data Management and Analysis}

Data were entered and cleaned by Epi Info version 3.5.1 and exported to SPSS version 20.0 for analysis. Descriptive statistics were done to calculate and present the data as frequencies and proportions with corresponding 95\% CI. Sensitivity, specificity, and level of agreement in slide readings were calculated using kappa analysis with kappa coefficient interpretations of $<0.20$ slight, $0.21-0.40$ fair, 0.41-0.60 moderate, 0.61-0.80 substantial, and 0.810.9 almost perfect agreement. Multivariate logistic regression with $p$-value $<0.05$ was done to identify factors affecting the performance of malaria laboratory diagnosis.

\section{Results}

\section{Characteristics of Laboratory Professionals and Quality Assurance Related Variables in Study Hospitals by Onsite Evaluation}

A total 46 laboratory professionals were included in the study. For about $60.9 \%$ of laboratory professionals, their educational status was degree followed by diploma. Of the laboratory professionals, $25(54.3 \%)$ had more than five years of service in malaria microscopy diagnosis. Thirty $(65.2 \%)$ had taken in-service training on malaria microscopy diagnosis. Of the total of 46 study health facilities, most of them had SOP, 39 (84.78\%), malaria guidelines, 37 (80.43\%), standard registration book, 32 (69.6\%), and request paper, 34 (73.9\%) in their laboratories. Thirty-four (73.9\%) of public hospital laboratories participated in the EQA program. The majority of public hospital laboratories performed QC regularly. Of the total studied health facilities, $60.9 \%$ of both thick and thin blood films were performed on the same slide and Giemsa stain reagent was the most used stain, 40 (89.95\%). Thirty-two (69.6\%) scanned a minimum of 100 fields to report negative results. However, $24(52.2 \%)$ of them did not report parasite density for positively detected parasites (Table 1).

\section{Misdiagnosed Results from Panel Test Slides}

From a total of 460 slides, 321 were discordant or misdiagnosed in the detection of parasites, species identification, and parasite density/count. Of 321 misdiagnosed slides, $85(26.47 \%)$ of them were discordant or misdiagnosed in species identification, 93 (29\%) of them were misdiagnosed in stage identification, $107(33.33 \%)$ of them were reported with an error in parasite density count, and 36 slides were reported with an error in detection of parasites. From the total that had discordant or misdiagnosed detection of parasites, $13(4.05 \%)$ and 23 $(7.17 \%)$ were false positive and false negative, respectively. Of the total of species misdiagnosis, $24(28,2 \%)$ slides were reported mixed as $P F$ followed by $P V$ as $P F 20$ (23.5\%). The most misdiagnosed stage identification was mixed stage reported as trophozoite (37.62\%) followed by mixed stage reported as gametocyte (21.5\%) (Table 2).

\section{Sensitivity, Specificity, and Agreement of Laboratory Professionals in Detecting Malaria Parasites of Validated PT Slides}

The overall sensitivity, specificity, PPV, and NPV were $77.63 \%, 96.41 \%, 97.82 \%$, and $85.23 \%$, respectively. The overall percent agreement in the detection of parasites was $80.45 \%$ with substantial agreement of kappa value (0.792). Laboratory professionals with $>5$ years of work experience had high sensitivity (78.33\%), specificity (98.3\%), and percent agreement (81.95\%) with substantial agreement 
Table I Laboratory Professionals and Quality Assurance Related Characteristics by Onsite Evaluation Checklist in Public Health Facilities of Eastern and Central Part of Oromia, Ethiopia, 2019

\begin{tabular}{|c|c|c|}
\hline Variables & Frequency & Percentage \\
\hline \multicolumn{3}{|l|}{ Qualifications } \\
\hline Diploma & 14 & $30.4 \%$ \\
\hline Degree & 28 & $60.9 \%$ \\
\hline Masters & 4 & $8.7 \%$ \\
\hline \multicolumn{3}{|l|}{ Service year } \\
\hline$<5$ years & 25 & $54.3 \%$ \\
\hline$>5$ years & 21 & $45.7 \%$ \\
\hline \multicolumn{3}{|l|}{ Training status } \\
\hline Trained & 30 & $65.2 \%$ \\
\hline Untrained & 16 & $34.8 \%$ \\
\hline \multicolumn{3}{|l|}{ Availability of SOP } \\
\hline Available & 39 & $84.78 \%$ \\
\hline Not available & 7 & $15.22 \%$ \\
\hline \multicolumn{3}{|c|}{ Availability of malaria guideline } \\
\hline Available & 37 & $80.43 \%$ \\
\hline Not available & 9 & $19.57 \%$ \\
\hline \multicolumn{3}{|c|}{$\begin{array}{l}\text { Availability of standard } \\
\text { registration book }\end{array}$} \\
\hline Available & 32 & $69.6 \%$ \\
\hline Not available & 14 & $30.4 \%$ \\
\hline \multicolumn{3}{|c|}{ Standard request paper } \\
\hline Available & 34 & $73.9 \%$ \\
\hline Not available & 12 & $26.1 \%$ \\
\hline \multicolumn{3}{|c|}{ Participate in EQA program } \\
\hline Participate & 34 & $73.9 \%$ \\
\hline Not participate & 12 & $26.1 \%$ \\
\hline \multicolumn{3}{|c|}{ Quality control performance } \\
\hline Regular & 32 & $69.65 \%$ \\
\hline Not regular & 14 & $30.35 \%$ \\
\hline \multicolumn{3}{|l|}{ Type of blood film } \\
\hline Thick & 17 & $37 \%$ \\
\hline Thin & I & $2.2 \%$ \\
\hline Both thick and thin & 28 & $60.9 \%$ \\
\hline \multicolumn{3}{|l|}{ Staining reagents used } \\
\hline Wright stain & 6 & $10 . \%$ \\
\hline Giemsa stain & 40 & $89 . \%$ \\
\hline \multicolumn{3}{|c|}{$\begin{array}{l}\text { Field scan to report negative } \\
\text { result }\end{array}$} \\
\hline Less than twenty five & 2 & $4.3 \%$ \\
\hline Fifty & 6 & $13 \%$ \\
\hline One hundred & 32 & $69.6 \%$ \\
\hline Two hundred & 6 & $13 \%$ \\
\hline
\end{tabular}

(Continued)
Table I (Continued)

\begin{tabular}{|l|l|l|}
\hline Variables & Frequency & Percentage \\
\hline $\begin{array}{l}\text { Parasite density reported if } \\
\text { needed } \\
\text { Yes }\end{array}$ & & \\
No & 22 & $47.8 \%$ \\
\hline $\begin{array}{l}\text { Slide validation by another } \\
\text { person before result release } \\
\text { Yes }\end{array}$ & 24 & $52.2 \%$ \\
No & 26 & \\
\hline
\end{tabular}

(kappa $=0.782$ ) compared to those who had work experience $\leq 5$ years in detecting malaria parasites. Laboratory professionals who were trained in malaria microscopy diagnosis had also greater sensitivity $(81.7 \%)$, specificity (98.3\%), and percent agreement (83.46\%) with almost perfect agreement (kappa $=0.85)$ in detecting malaria parasite compared to those who were not trained (Table 3).

\section{Factors Affecting Quality of Malaria Microscopy Diagnosis in Public Hospitals Laboratories}

Multivariate analysis was done to evaluate and identify factors that affect the quality of malaria microscopy diagnosis. Based on the percent agreement as a dependent variable, work experience and training status were significantly associated with the quality of malaria microscopy diagnosis. Laboratory professionals who had work experience greater than five years were 10.56 times better in detecting and identifying malaria parasites when compared to those who had work experience less than or equal to five years (AOR $[95 \% \mathrm{CI}]=10.56[1.45-76.73]$ ). Laboratory professionals who were trained in malaria microscopy diagnosis reported 6.12 times better quality results than those who were not trained (AOR, \% 95CI=1.28 [1.5-48.13]) (Table 4).

\section{Discussion}

Evaluation of malaria microscopy diagnosis performance is essential to ensure accurate detection and identification of malaria parasites in human blood. In this study, factors affecting the quality of malaria microscopy diagnosis in the health facilities were assessed. From the total of 46 hospitals included in the study, 31 (67.39\%) had an acceptable quality performance of malaria diagnosis with cumulative grading score of $>80 \%$, which was determined from 
Table 2 Misdiagnosed Slide Result Reports of Health Facilities' Laboratory Professionals in Species Identification, Stage Identification, Parasite Density and Detection of Parasite from Distributed Panel Test Slides in Easter and Central Part of Oromia Region, Ethiopia, 2019

\begin{tabular}{|c|l|l|}
\hline Variables & $\begin{array}{l}\text { Frequency } \\
\text { (\%) }\end{array}$ & $\begin{array}{l}\text { Percentage } \\
\text { (\%) }\end{array}$ \\
\hline $\begin{array}{l}\text { Species misdiagnosis (N=85) } \\
\text { PV reported as PF }\end{array}$ & 20 & \\
PF reported as PV & 11 & $23.5 \%$ \\
PF as mixed & 6 & $12.94 \%$ \\
PV as mixed & 7 & $7.1 \%$ \\
Mixed reported as PF & 24 & $8.2 \%$ \\
Mixed reported as PV & 8 & $28.2 \%$ \\
Pf as negative & 2 & $9.4 \%$ \\
PV as negative & 7 & $2.3 \%$ \\
\hline Stage misidentification (N=93) & & $8.2 \%$ \\
Gametocyte as trophozoite & 7 & $17.2 \%$ \\
Trophozoite as gametocyte & 9 & $7.51 \%$ \\
Trophozoite as mixed & 16 & $9.67 \%$ \\
Gametocyte as mixed & 6 & $17.2 \%$ \\
Mixed as trophozoite & 35 & $6.5 \%$ \\
Mixed as gametocyte & 20 & $37.62 \%$ \\
\hline Parasite density miscounted & 107 & $21.5 \%$ \\
(N=107) & & $33.3 \%$ \\
\hline Misdetection of parasites & & \\
(N=36) & & \\
False positive & 13 & $7.16 \%$ \\
False negative & 23 & $100 \%$ \\
\hline Total discordant slides & 321 & \\
\hline & & \\
\hline
\end{tabular}

the points given based on nationally established evaluation criteria: positive reported as positive, negative reported as negative, correct species identification, correct stage identification, and correct parasite count for 10 slides distributed to each facility. According to ISO 15,189, quality and competence requirements facility which scored $>80 \%$ has good quality performance of any laboratory diagnosis. ${ }^{3,24}$

The average quality of malaria diagnosis in this study was good, which was not similar to the studies done in West Oromia and Pakistan. ${ }^{23,25}$ The possible variation might be a lack of training and the difference in study health facilities which in this study were based in hospitals only, where there was high manpower, in contrast to the two studies which were based in health centers. This might affect the sensitivity and specificity of the laboratory professionals in reporting good results.

The overall percent agreement of laboratory professionals in detection and species identification was $80.45 \%$ (kappa $=0.79)$ and $63.03 \%(k a p p a=0.38)$ with a substantial and fair agreement based on kappa index interpretation. ${ }^{27}$ This study was consistent with the study done in Tanzania and Tigray, North Ethiopia in which the level of agreement in detection was substantial, with percent of agreement $87 \%$ (kappa $=0.74)$ and $79 \%$ (kappa $=0.62)$, respectively, and fair agreement in species identification in the study conducted in Ethiopia with percent agreement of $64.77 \%(\mathrm{kappa}=0.33){ }^{21,27}$

However, this study was not supported by the study done in Kenya from non-quality assurance piloted program facilities in which level of agreement in the detection of the parasite was fair with a percent agreement of $77 \%$ (kappa $=0.35$ ). The possible reason was the facilities included in Kenya were only from non-quality assurance pilot enrolled facilities, in contrast to this study. ${ }^{20}$

In this study, the overall discordant rate in detection and species identification of the total distributed and examined slides was $26 \%$. This was higher than in the studies in West Amhara and Pakistan, which were $6.75 \%$, and $0.5-1 \%$, respectively, and lower than the study done in Addis Ababa which showed $39.5 \%{ }^{25,28}$ The discrepancy might be due to the difference in method and frequency of assessment in

Table 3 Overall Sensitivity, Specificity, and Percent Agreement of Health Facilities Laboratory Professionals in Detecting Malaria Parasites from Distributed Proficiency Test Slides

\begin{tabular}{|c|c|c|c|c|c|c|}
\hline \multirow[t]{2}{*}{ Variables } & Sensitivity & Specificity & PPV & NPV & \%agreement & Kappa \\
\hline & $77.63 \%$ & $96.41 \%$ & $97.82 \%$ & $85.23 \%$ & $80.45 \%$ & 0.792 \\
\hline \multicolumn{7}{|l|}{ Work experience } \\
\hline$>5$ years & 78.33 & 98.3 & 100 & 86.79 & 81.95 & 0.782 \\
\hline$<5$ years & 77.04 & 94.76 & 96 & 83.908 & 79.2 & 0.689 \\
\hline \multicolumn{7}{|l|}{ Training status } \\
\hline Trained & 81.7 & 98.3 & 99.33 & 89.29 & 83.46 & 0.85 \\
\hline Untrained & 70 & 92.87 & 95 & 77.60 & 74.81 & 0.682 \\
\hline
\end{tabular}

Abbreviations: PPV, positive predictive value; NPV, negative predictive value. 
Table 4 Multivariate Analysis for Factors Affecting Quality of Malaria Microscopic Diagnostic Performance

\begin{tabular}{|c|c|c|c|c|c|c|}
\hline Variables & Freq (\%) & COR & $95 \% \mathrm{Cl}$ & AOR & $95 \% \mathrm{Cl}$ & $P$-value \\
\hline \multicolumn{7}{|l|}{ Work experience } \\
\hline$>5$ year & 21 & I & & I & & \\
\hline$<5$ year & 25 & 8.56 & $1.97-9.35$ & 10.56 & $1.45-76.73$ & $0.020 *$ \\
\hline \multicolumn{7}{|l|}{ Training status } \\
\hline Trained & 30 & 1 & & I & & \\
\hline Not trained & 16 & 4.12 & $\mathrm{I}, 43-6.45$ & 6.12 & $1.5-48.13$ & $0.032 *$ \\
\hline \multicolumn{7}{|l|}{ QC* performance } \\
\hline Regular & 32 & I & & I & & \\
\hline Not regular & 14 & 0.33 & $0.803-11.21$ & 0.592 & $0.09-3.98$ & 0.590 \\
\hline \multicolumn{7}{|c|}{ Slide rechecked when result released } \\
\hline Yes & 26 & I & & I & & \\
\hline No & 20 & 4.20 & $1.132-15.58$ & 0.360 & $0.06-2.32$ & 0.284 \\
\hline \multicolumn{7}{|l|}{ Type of blood film } \\
\hline Both thick and Thin & 29 & I & & I & & \\
\hline Thick film only & 17 & 2.4 & $0.68-8.45$ & 5.664 & $0.65-49.70$ & 0.118 \\
\hline \multicolumn{7}{|l|}{ Availability of SOP* } \\
\hline Available & 39 & I & & I & & \\
\hline Not available & 7 & 7.25 & $1.21-43.44$ & 0.992 & I.I-9.95 & 0.994 \\
\hline
\end{tabular}

Note: *Significantly associated variables with $p$-value $<0.05=$ work experience and training status.

Abbreviations: $\mathrm{COR}$, crude odd ratio; $\mathrm{Cl}$, confidence interval; $\mathrm{AOR}$, adjusted odd ratio; SOP, standard operating procedure; QC, quality control.

which studies were conducted for more than one round in Bahir Dar and Pakistan, compared to this study.

From the total of discordant slides, false positivity and false negativity rate were $9.5 \%$ and $5.6 \%$, which implies either wrongly considering artifacts as parasites in smears due to poor quality of reagent or incorrect staining procedure, or failure to scan the recommended field to detect a low concentration of parasites and lack of training. This study is supported by the study done in West Amhara and Addis Ababa in which false positivity rates ranged from $0.79 \%$ to $10.5 \%{ }^{15,22}$

This study reported lower false-positive rate than the study done in Democratic Republic of Congo and West Oromia in which false positivity rate results were $24.6 \%$ and $24.4 \% .{ }^{16,24}$ This indicates the laboratory professionals incorrectly considered artifacts as parasites, which leads to mistreatment of suspected malaria patients and delayed diagnosis of the underlying cause of the illness.

The overall sensitivity and specificity of laboratory professionals in the detection of malaria parasites were $77.63 \%$ and $96.41 \%$, respectively. These findings were similar in the studies conducted in Hawassa and Bahir Dar city in which sensitivity and specificity were $82 \%$ and $96.2 \%, 83 \%$ and $97 \%$, and $88 \%$ and $97 \%$, respectively. ${ }^{28,29}$ The lower sensitivity indicates true positives were missed, which leads to the exposure to unnecessary toxic drugs and delays in treatment. However, these findings were not supported by the study done in Tanzania in which higher sensitivity and lower specificity were revealed, with $84.3 \%$ and $90.8 \%{ }^{27}$. The variations might be the difference in the assessment method and the implementation of the quality assurance program.

In this study, work experience was significantly associated with the quality of malaria microscopy diagnosis. Laboratory professionals who had work experience of more than five years reported 10.56 times more good results than those who had work experience of less than five years. This study is in line with the study conducted in Kenya in which laboratory professionals who had more work experience diagnosed malaria 3.8 times more accurately than those with less work experience. ${ }^{22}$

However, this study is not similar to the study done in Tigray, North Ethiopia, where work experience in malaria diagnosis is not significantly associated with quality of malaria diagnosis. ${ }^{21}$ The discrepancy might be due to the method of data collection and sampling technique used in the study done in Tigray. 
In-service training was another factor significantly associated with the quality of malaria microscopy performance in this study. Laboratory professionals who were trained in malaria microscopy diagnosis reported 6.12 times better quality results than those who were not trained.

This study is consistent with the study done in Tigray, West Oromia, Addis Ababa, and Democratic Republic of Congo in which training status of laboratory professionals showed significant association with quality of malaria microscopy diagnosis. ${ }^{14,21-23}$ This might show that provision of theoretical and practical refresher training for laboratory personnel is a primary means of accurate detection and identification of malaria parasites.

\section{Limitation of the Study}

Although External Quality Assessment was used to evaluate the performance of malaria microscopy diagnosis, slides were not collected from all study hospitals for blind rechecking because of a lot of gaps including insufficient slide boxes for proper storage of slides at health facilities. Despite the discordance in stage identification and parasite density, percent and level of agreement was not calculated for both.

\section{Conclusions}

In this study, the overall percent agreement of laboratory professionals in detection and species identification was $80.45 \%$ and $63.03 \%$, with substantial and fair agreement, respectively. The overall sensitivity and specificity of laboratory professionals in detection of malaria parasites were $77.63 \%$ and $96.41 \%$, respectively. Lack of training and low work experience of laboratory professionals were factors associated with malaria microscopy diagnostic performance. Hence, this study indicated that capacitating laboratory professionals is essential to ensure good performance of malaria microscopy, which reduces misdiagnosis of malaria parasites and of malaria suspected patients.

\section{Abbreviations}

EQA, External Quality Assessment; WHO, World Health Organization; RDT, Rapid Diagnostic Test; FMOH, Federal Ministry of Health; QA, Quality Assurance; PT, proficiency test; SOP, Standard Operating Procedure; ISO, International Standard Organization; PF, Plasmodium falciparum; PV, Plasmodium vivax.

\section{Ethical Approval and Consent to Participate}

Ethical clearance was obtained from the Oromia Regional Health Bureau Ethical review board. The Oromia Regional Health Bureau Ethical Review Board Reviewed the research protocol and granted full approval internalizing the existing problem and the study eventually come with possible solution. A formal letter was also written to each hospital by Oromia regional health bureau ethics review committee in compliance with the National Health Research ethics review committee so that the confidentiality of the participants could be ensured. All laboratory professionals who participated in the study were provided informed consent in writing, so that their rights and interests were protected, ensuring the study was in accordance with the Declaration of Helsinki.

\section{Acknowledgments}

We would like to acknowledge Adama Public Health Research and Referral Laboratory Center for resource support in data collection. We also extend our special thanks to the Oromia Regional Health Bureau Ethics review committee, Department of Public Health Emergency Preparedness and Research who provided us ethical clearance and laboratory professionals at study hospitals for timely examination and report of distributed proficiency test slide results.

\section{Author Contributions}

All authors made substantial contribution to the conception and design, acquisition of data, analysis and interpretation of data; took part in drafting, revising and critically reviewing the article; gave final approval of the version to be published; have agreed on the journal to which the article has been submitted and are accountable for all aspects of the work.

\section{Disclosure}

The authors declare that they have no competing interests.

\section{References}

1. World Health Organization Guideline for the Treatment of Malaria 3rd Ed. Swizerland, Geneva; 2015.

2. National Malaria Diagnosis Quality Assurance Guidelines National Department of Health South Africa. 2011.

3. Malaria Laboratory Diagnosis External Quality Assessment Scheme Guidelines Malaria Laboratory Diagnosis External Quality Assessment Scheme Guidelines. 3rd. Federal republic of Ethiopia Ministry of health; 2017. 
4. WHO external quality assurance scheme for malaria nucleic acid amplification testing Operational manual. December, 2017. Available from: https://www.who.int/malaria/publications/atoz/ NAAT-EQA-manual/en/. Accessed August 1, 2020.

5. World health organization. Global technical strategy for malaria 2016-2030. 2016.

6. World malaria report. 2018. Available from: https:/www.who.int/ malaria/publications/world-malaria-report-2018/en/. Accessed August $1,2020$.

7. World malaria report. 2017. Available from: https:/www.who.int/ malaria/publications/world-malaria-report-2017/report/en/. Accessed August 1, 2020.

8. Years N, Quality I, Services C. Malaria laboratory diagnosis and nine years of action for improving quality and case management service $\mathrm{s}$ in Ethiopia. Malaria J. 2016;15:1-8.

9. Guidelines for case management of malaria. 3rd. Ghana: Ministryof health; July, 2014. Available from: https://www.ghanahealthservice. org/downloads/GUIDELINE\%20FOR\%20CASE\%20MANAG MENT\%20.pdf. Accessed August 1, 2020.

10. Bailey JW, Williams J, Bain BJ, Parker-williams J, Chiodini PL. Guideline: the laboratory diagnosis of malaria. $\mathrm{Br} J$ Haematol 2013;163:573-580.

11. Adugna F, Deremo B, Emmet W, et al. Evaluation USAID/Ethiopia Midterm Evaluation of the Malaria Laboratory Diagnosis and Monitoring Project; December 2015.

12. Taffese HS, Hemming-schroeder E, Koepfli C, et al. Malaria epidemiology and interventions in Ethiopia from 2001 to 2016. Infect Dis Poverty. 2018;7(1):1-9. doi:10.1186/s40249-018-0487-3

13. World Health Organization Malaria microscopy quality assurance manual. Vol.1. 2009:2009.

14. Mukadi P, Lejon V, Barbé B, Gillet P, Nyembo C. Performance of microscopy for the diagnosis of malaria and human African trypanosomiasis by diagnostic laboratories in the democratic Republic of the Congo: results of a Nation-Wide External Quality Assessment. PLoS One. 2016;11(1):e0146450.

15. Hailu HA, Shiferaw MB, Demeke L, et al. External quality assessment of malaria microscopy diagnosis among public health facilities in West Amhara Region, Ethiopia. BMC Res Notes. 2017;1-5. doi:10.1186/s13104-017-3080-0

16. Ministry of Health and sport. Quality Assurance and Quality Control Manual for Malaria Microscopy Version.2. Myanmar; 2017.
17. World Health Organization Global malaria Programme. Malaria case management Operation Manual. 2009. Available from: https://www. who.int/malaria/publications/atoz/9789241598088/en/. Accessed August 1, 2020.

18. World Health Organization. Malaria microscopy quality assurance manual-version 2. 2016.

19. Annual Oromia regional statical report. Oromia. 2019.

20. Odhiambo F, Buff AM, Moranga C, et al. Factors associated with malaria microscopy diagnostic performance following a pilot quality - assurance programme in health facilities in malaria low - transmission areas of Kenya, 2014. Malar J. 2017;2018:1-10.

21. Alemu M, Tadesse D, Hailu T, et al. Performance of laboratory professionals working on malaria microscopy in Tigray, North Ethiopia. J Parasitol Res. 2017;2017.

22. Gebrekidan LD, Hiruy HN. Assessment of malaria microscopic diagnosis performance of laboratory professionals in Addis Ababa' $\mathrm{s}$ public health facilities. Training. 2019;5(1):1-6.

23. Sori G, Zewdie O, Tadele G, Samuel A. External quality assessment of malaria microscopy diagnosis in selected health facilities in Western Oromia, Ethiopia. Malar J. 2018;1-7. doi:10.1186/s12936018-2386-2

24. WHO-AFRO Laboratory Accreditation Assessment. Check list for clinical and public health laboratories- ISO 15189 document requirements for quality and competence. 2012.

25. Khan MA, Walley JD, Munir MA, Khan MA. District level external quality assurance (EQA) of malaria microscopy in Pakistan: pilot implementation and feasibility. Malar J. 2011;10(1):45.

26. Landis GR, Koch GG. A one way component of variance model for categorical data. Biometrics. 1977;33:671-679. doi:10.2307/2529465

27. Ngasala B, Bushukatale S. Evaluation of malaria microscopy diagnostic performance at private health facilities in Tanzania. Malar J. 2019;1-7. doi:10.1186/s12936-019-2998-1

28. Atalele K, Id J, Melaku MY, Jemeber TH. Performance evaluation of laboratory professionals on malaria microscopy at health facilities in Bahir Dar city administration, Northwest Ethiopia. PloS One 2018;110.

29. Ayalew F, Tilahun B, Taye B. Performance evaluation of laboratory professionals on malaria microscopy in Hawassa. BMC Res Notes. 2014;7(1):839.
Pathology and Laboratory Medicine International

\section{Publish your work in this journal}

Pathology and Laboratory Medicine International is a peer-reviewed, open access journal focusing on innovative basic research and translational research related to pathology or human disease. The journal includes original research, updates, case reports, reviews and commentaries on current controversies. The manuscript management system is completely online and includes a very quick and fair peer-review system. Visit http://www.dovepress.com/testimonials.php to read real quotes from published authors. 